\title{
Efecto de la interacción del hongo micorrícico arbuscular (AMF) Glomus intraradices y Trichoderma harzianum sobre la producción de plantines de melón en zonas áridas
}

\author{
Interaction effects of arbuscular mycorrhizal fungi $(A M F)$ and Glomus intraradices \\ and Trichoderma harzianum on melon seedling production in arid
}

\author{
Christian Santander ${ }^{1,2 *}$, Jorge Olave $e^{1,2}$
}

\begin{abstract}
RESUMEN
Esta investigación fue realizada en un invernadero semicontrolado de la Estación Experimental Canchones, donde se evaluó el efecto interactivo del hongo micorrícico arbuscular (AMF) Glomus intraradices y Trichoderma harzianum (Th) sobre los parámetros de crecimiento, diferenciación, indicadores de estrés y bioquímicos en plántulas de melón; así como también el porcentaje de micorrización y unidades formadoras de colonia de Th (UFC). El material vegetal utilizado correspondió a melón cv. Honeydew tipo Inodorus. Los tratamientos evaluados fueron: control $\left(\mathrm{T}_{0}\right)$, inoculación con GI en dosis de 40 esporas * planta ${ }^{-1}\left(\mathrm{~T}_{1}\right)$, inoculación con GI en dosis de 40 esporas planta ${ }^{-1}+1,5 * 10^{8}$ UFC de Th bandeja $^{-1}\left(\mathrm{~T}_{2}\right)$ e inoculación con Th en dosis $1,5 * 10^{8}$ bandeja $^{-1}$ $\left(\mathrm{T}_{3}\right)$. La inoculación con GI se realizó en el momento de la siembra y la inoculación con Th se realizó 15 días después. Se utilizó un diseño completamente aleatorizado realizándose un análisis de varianza multifactorial (ANOVA) y para la separación de medias se utilizó el test LSD, mediante el programa estadístico INFOSTAT a un $\alpha=0,05$. Los resultados obtenidos 50 días después de la siembra determinaron que la coinoculación con GI $+\mathrm{Th}\left(\mathrm{T}_{2}\right)$ disminuyó el porcentaje de micorrización por una acción antagónica de Th sobre GI, comparándolo con el mayor porcentaje de micorrización determinado en $\mathrm{T}_{1}$, en los tratamientos $\mathrm{T}_{0}$ y $\mathrm{T}_{3}$ no se determinó colonización micorrícica. En cuanto a los tratamientos inoculados con $\mathrm{Th}_{\mathrm{C}}\left(\mathrm{T}_{2}\right.$ y $\left.\mathrm{T}_{3}\right)$, no se determinaron diferencias significativas entre los tratamientos inoculados, pero sí se determinó una disminución en la población de Th entre los días 1 y 50. La inoculación solo con GI presentó mayor producción de biomasa radical, mayor relación peso seco raíz : peso seco vástago y menor índice de ahilamiento. La actividad nitrato reductasa (NRI/NRE) fue afectada solo en los tratamientos inoculados con GI, determinándose una mayor relación en estos tratamientos. La coinoculación entre GI y Trichoderma no determinó un efecto como promotor de crecimiento en las plantas de melón; es más, Trichoderma tuvo un efecto detrimental sobre la colonización micorrícica. Al contrario, GI inoculado de forma individual tuvo un efecto sobre el crecimiento radicular, lo que está determinado por el alto nivel de colonización.
\end{abstract}

Palabras clave: Glomus intraradices, Trichoderma harzianum, Cucumis melo, semilleros.

\begin{abstract}
This research was performed in a semi-controlled greenhouse at the Canchones Experimental Station, where the interactive effect of arbuscular mycorrhizal fungi (AMF) Glomus intraradices (GI) and Trichoderma harzianum (Th) on the parameters of growth, differentiation, stress indicators and biochemical parameters, as well as the percentage of mycorrhization and colony forming units $T h(C F U)$. The plant material used corresponded to melon cv. Honeydew Inodorus type. The treatments were: control $\left(T_{0}\right)$, inoculation with GI at doses of 40 spores per plant $\left(T_{1}\right)$, inoculation with GI at doses of 40 spores per plant $+1.5 * 10^{8}$ CFU of Th per tray $\left(T_{2}\right)$ and Th dose inoculation with $1.5 * 10^{8}$ per tray $\left(T_{3}\right)$. Inoculation with GI was performed at the time of sowing and inoculation with Th was performed 15 days later. We used a design performing completely randomized multifactorial variance analysis (ANOVA) and mean separation test was used LSD, using the statistical software INFOSTAT $\alpha=0.05$. The results obtained in the destructive sampling at 50 days of sowing determined that co-inoculation with $G I+T h\left(T_{2}\right)$ decreased the percentage of mycorrhizal colonization by an antagonistic action of Th on GI, compared with the highest percentage of mycorrhizal determined in $T_{1}$, in $T_{0}$ and $T_{3}$ treatments not mycorrhizal colonization was determined. As for the treatments inoculated with $T h\left(T_{2}\right.$ and $\left.T_{3}\right)$, no significant differences between treatments were determined inoculated, but a decrease in the population of Th between days 1 and 50 was determined. The only inoculation with GI had higher root biomass production, higher root dry weight ratio: stem dry weight and lower rate of etiolation. The nitrate reductase (NRI/NRE) activity was affected only in the treatments inoculated with GI, determining a higher ratio in these treatments. Co-inoculation between AMF and Trichoderma not determine an effect in promoting growth in melon plants, is Trichoderma had a detrimental effect on mycorrhizal colonization. Unlike GI inoculated individually had an effect on root growth which is determined by the high level of colonization.
\end{abstract}

Key words: Glomus intraradices, Trichoderma harzianum, Cucumis melo, seedling.

\footnotetext{
1 Centro de Investigación y Desarrollo en Recursos Hídricos, Iquique, Chile.

2 Universidad Arturo Prat, Casilla 121, Iquique, Chile.

* Autor para correspondencia: christian.santander@ ciderh.cl
} 


\section{Introducción}

El suelo es un sistema complejo donde habita una gran diversidad de especies vegetales, animales y microbianas, estableciendo relaciones entre sus componentes de formas variadas y complejas, lo que contribuye a clasificar a los suelos con características propias mediante la modificación de las fases sólida, líquida y gaseosa (Ben-Omar et al., 1997). En zonas áridas y semiáridas el suelo presenta valores bajos en materia orgánica por la escasa cubierta vegetal y limitada productividad, siendo la materia orgánica fundamental para generar buenas condiciones de establecimiento para las plantas (Chapin et al., 1996). Por ello, la rizosfera debe constituirse en la zona donde se presente la mayor actividad microbiana para la síntesis de promotores de crecimiento de las plantas (Azcón-Aguilar et al., 2002). Según Paul y Clark (1989), los microorganismos tienen gran importancia en las características edáficas de los suelos; ciclos biogeoquímicos de elementos como el carbono, nitrógeno, oxígeno, azufre, fósforo y hierro, fertilidad de las plantas y protección frente a patógenos; degradación de compuestos xenobióticos y producción de fitohormonas. Avis et al. (2008) señalan que los microorganismos del suelo desempeñan una función importante en el mantenimiento de la estabilidad de agrosistemas, contribuyendo a la fertilidad del suelo, a la estructura y biodiversidad y tienen un real efecto sobre el crecimiento y desarrollo de las plantas.

Barea y Azcón-Aguilar (1982) clasifican a los microorganismos según sus relaciones con las plantas diferenciándolos en simbiontes parasíticos o "patógenos", causantes de enfermedades a las plantas; simbiontes mutualistas, los cuales benefician el desarrollo y nutrición vegetal y microorganismos saprófitos, los cuales obtienen su fuente nutricional a partir de compuestos orgánicos procedentes de residuos animales, vegetales o microbianos. Además existen dos grandes grupos de microorganismos de interés agrícola: Promotores del Crecimiento Vegetal (PGPM) y Agentes de Control Biológico (BCA) (Green et al., 1999). En el grupo de los PGPM se identifican efectos primarios, como la síntesis de hormonas que estimulan el crecimiento, solubilización de nutrientes con independencia de la acidez del suelo aumentando su disponibilidad para las plantas y mayor tolerancia a estrés abiótico (Yasir et al., 2009). Entre los efectos secundarios, el más relevante es el control de enfermedades (Aliye et al., 2008). En cambio, el grupo BCA se identifica a Trichoderma y Pseudomonas sp. (Harman, 2006), entre otros microorganismos biocontroladores. Destaca el parasitismo y la inhibición del crecimiento de fitopatógenos como efectos primarios; y entre los efectos secundarios se ha demostrado un efecto estimulante en el crecimiento de las plantas, degradación de la materia orgánica y aumento en la disponibilidad de nutrientes (Avis et al., 2008).

En la rizosfera se han encontrado interacciones neutras, positivas y negativas (Azcón-Aguilar et al., 2002). Calvet et al. (1993) registraron efectos sinérgicos entre Trichoderma y hongos micorrícicos arbusculares sobre el crecimiento de Tagetes erecta. Sin embargo, McAllister et al. (1994) observaron efectos detrimentales en la producción de biomasa. A partir de estos antecedentes, el objetivo de este estudio fue evaluar la interacción de un hongo micorrícico arbuscular y Trichoderma harzianum sobre parámetros de crecimiento, diferenciación, indicadores de estrés y bioquímicos en plántulas de melón.

\section{Materiales y Métodos}

La investigación se llevó a cabo en el invernadero semiclimatizado de la Estación Experimental Canchones, con una duración de 50 días desde la siembra de semillas de melón Honey Dew cv. Orange tipo Inodorus hasta el tercer muestreo de los plantines. La inoculación con GI se realizó con el producto comercial Biosim ${ }^{\circledR}$, el que contiene esporas en latencia de Glomus intraradices, la dosis utilizada fue de 40 esporas planta ${ }^{-1}$, y la inoculación con Trichoderma harzianum se realizó con el producto comercial Tricho- $\mathrm{D}^{\circledR}$, a una dosis de 1,5 $* 10^{8}$ UFC 3 L de sustrato ${ }^{-1}$. La siembra se realizó en bandejas de plástico termoformado de 72 celdas de un volumen por celda de $43 \mathrm{ml}$, utilizándose una mezcla de 70:30\% de Turba + Perlita.

El manejo realizado consistió en aportar mediante el riego solo agua desde la emergencia de los cotiledones hasta la primera hoja verdadera (Tabla 1). A partir de esta fase hasta la segunda hoja verdadera se aplicó $1 / 3$ de la disolución de fertirriego, hasta la tercera hoja verdadera $2 / 3$ y en la última etapa la concentración total (Tabla 2). La disolución nutritiva se ajustó a un pH entre 6,0 a 6,5; una $\mathrm{CE}$ final de $1,6 \mathrm{dS} \mathrm{m}^{-1}$ equivalente a un potencial osmótico ( $\Psi \mathrm{s})$ de -0,067 MPa. Se midió el pH y la CE, con una frecuencia de tres días 
Tabla 1. Calidad química del agua de riego de la Estación Experimental Canchones ${ }^{1}$.

\begin{tabular}{cccccccccccc}
\hline \multirow{2}{*}{$\mathrm{pH}$} & \multirow{8}{*}{$\begin{array}{c}\mathrm{CE} \\
\mathrm{dS} \mathrm{m}{ }^{-1}\end{array}$} & $\mathrm{HNO}^{3-}$ & $\mathrm{NO}_{3}$ & $\mathrm{Cl}^{-}$ & $\mathrm{SO}_{4}=$ & $\mathrm{K}^{+}$ & $\mathrm{Ca}^{+2}$ & $\mathrm{Mg}^{+2}$ & $\mathrm{Na}^{+}$ & $\mathrm{NH}^{4+}$ \\
\hline 7,57 & 0,8 & 1,6 & 0,07 & 3,25 & 2,7 & 0,19 & 3,54 & 0,12 & 4,07 & 0,06 \\
\hline
\end{tabular}

${ }^{1}$ Agua subterránea. AGROLAB (2011).

Tabla 2. Disolución (meq L ${ }^{-1}$ ) de fertirriego utilizadas en plántulas de melón Honeydew (tipo Inodorus).

\begin{tabular}{ccccccccc}
\hline Disoluciones & $\mathrm{H}_{2} \mathrm{PO}_{4}^{-}$ & $\mathrm{NO}_{3}^{-}$ & $\mathrm{SO}_{4}{ }^{-}$ & $\mathrm{K}^{+}$ & $\mathrm{Ca}^{2+}$ & $\mathrm{Mg}^{2+}$ & $\mathrm{NH}^{2+}$ \\
\hline $\mathrm{DN}^{1}$ & 1,25 & 7,50 & 2,50 & 3,25 & 5,00 & 2,50 & 0,5 \\
\hline
\end{tabular}

${ }^{1}$ Steiner modificado (Casas, 2005); Disolución ajustada con concentraciones de $\mathrm{Cl}^{-} \mathrm{y} \mathrm{Na}^{+}$presentes en aguas de riego salinas.

para efectuar los ajustes de frecuencia y tiempo de riego para no superar el valor máximo de $\mathrm{CE}$, equivalente al umbral de tolerancia para el cultivo de melón (Urrestarazu, 2004). Además, para mitigar el efecto de la salinidad en la rizosfera se ajustó la disolución nutritiva con balances iónicos entre $\mathrm{Na}^{+} / \mathrm{Ca}^{2+}$ de 0,81 y $\mathrm{Cl} / \mathrm{NO}_{3}{ }^{--}$de 0,43 . (Guzmán y Olave, 2006).

Los tratamientos evaluados fueron cuatro: control no inoculado $\left(\mathrm{T}_{0}\right)$, inoculación con GI a una dosis de 40 esporas planta ${ }^{-1}\left(\mathrm{~T}_{1}\right)$, inoculación con AMF en dosis de 40 esporas planta ${ }^{-1}+\mathrm{Th}$ en dosis $1,5 * 10^{8}$ UFC $3 \mathrm{~L}$ de sustrato $^{-1}\left(\mathrm{~T}_{2}\right)$, e inoculación con Th en dosis $1,5 * 10^{8}$ UFC $3 \mathrm{~L}$ de sustrato $^{-1}\left(\mathrm{~T}_{3}\right)$. La inoculación con GI se realizó en el momento de la siembra y la inoculación con Th se realizó 15 días después de siembra. Cada tratamiento correspondió a una bandeja y cada celda fue una repetición (72 celdas). Se realizó un muestreo destructivo de cinco plantas por cada tratamiento a los 30, 45 y 50 días postsiembra. En raíces se determinó el porcentaje de micorrización por el método descrito por Phillips y Hayman (1970) modificado, y posteriormente se calculó el grado de micorrización de las raíces mediante el método de Trouvelot (Trouvelot et al., 1986). En 1 gramo de sustrato se determinó el crecimiento poblacional de Th (UFC) mediante el método de las diluciones seriadas, siembra por extensión en placas de Petri con medio de cultivo selectivo propuesto por Williams et al. (2003) y recuento de colonias. En plantas se determinó diferenciación de tejidos, producción de biomasa, crecimiento e indicadores de estrés indirecto.

Se evaluó la actividad enzimática de la enzima nitrato reductasa endógena e inducida, como parámetro bioquímico. Para la actividad endógena se utilizó el método descrito por Bar-Akiva et al. (1970), adaptada por Valenzuela et al. (1987), y para la actividad inducida se utilizó el método de Bar-Akiva y Sternbaum (1966), modificado por BarAkiva et al. (1970) y adaptado por Valenzuela et al. (1987). La intensidad del color desarrollado se midió a una absorbancia de $540 \mathrm{~nm}$ en un espectrofotómetro (SPECTRONIC, modelo GENESYS 2). Las lecturas obtenidas de las muestras se llevaron a una recta de calibración en el rango de 0 a $4 \mu \mathrm{M}$, elaborada con $\mathrm{NaNO}^{-2}$, con una concentración de $1 \mathrm{mM}$ como solución patrón y por dilución volumétrica se realizaron las diferentes concentraciones entre el rango mencionado. Los resultados se expresaron en $\mu \mathrm{M}$ de $\mathrm{NO}^{-2} * \mathrm{~g}^{-1} \mathrm{pf} \mathrm{h}^{-1}$.

El diseño experimental utilizado fue completamente aleatorizado. Para los datos obtenidos se realizó análisis de varianza multifactorial, y para la separación de medias se utilizó el test LSD, mediante el software estadístico INFOSTAT, a un $\alpha \leq 0,05$. Para el número de hojas los datos se normalizaron para su análisis por medio de transformación logarítmica expresada por la ecuación $\log _{10}(\mathrm{X}+1)$, y los datos porcentuales se normalizaron con la transformación de Bliss expresada en la ecuación $\operatorname{arcos} \operatorname{arcos} \sqrt{x}$.

\section{Resultados y Discusión}

\section{Efecto de T. harzianum sobre la micorrización}

La coinoculación con el hongo micorrícico y $T$. harzianum en plántulas de melón presentó diferencias significativas en el porcentaje de micorrización (Tabla 3). El tratamiento 1 presentó una respuesta altamente significativa ( $\mathrm{p}$-valor $\leq 0,0001)$, con 
Tabla 3. Parámetros de producción de biomasa seca, porcentaje de micorrización y variación poblacional de Th en plántulas de melón Inodorus var. Honeydew a los 50 días de siembra.

\begin{tabular}{lcccccc}
\hline & \multicolumn{9}{c}{ Parámetros } \\
\cline { 2 - 7 } Tratamientos & $\begin{array}{c}\text { Micorrización } \\
(\%)\end{array}$ & $\begin{array}{c}\text { UFC } \\
\left(10^{7}\right)\end{array}$ & $\begin{array}{c}\text { MSHj } \\
(\mathrm{g})\end{array}$ & $\begin{array}{c}\text { MSTa } \\
(\mathrm{g})\end{array}$ & $\begin{array}{c}\text { MSRz } \\
(\mathrm{g})\end{array}$ & $\begin{array}{c}\text { MS Total } \\
(\mathrm{g})\end{array}$ \\
\hline $\mathrm{T}_{0}$ & $0 \mathrm{a}$ & $0 \mathrm{a}$ & 0,47 & 0,20 & $0,09 \mathrm{a}$ & 0,76 \\
$\mathrm{~T}_{1}$ & $49,8 \mathrm{c}$ & $0 \mathrm{a}$ & 0,49 & 0,17 & $0,21 \mathrm{c}$ & 0,87 \\
$\mathrm{~T}_{2}$ & $21,4 \mathrm{~b}$ & $7,35 \mathrm{~b}$ & 0,48 & 0,20 & $0,16 \mathrm{~b}$ & 0,84 \\
$\mathrm{~T}_{3}$ & $0 \mathrm{a}$ & $7,35 \mathrm{~b}$ & 0,54 & 0,25 & $0,10 \mathrm{a}$ & 0,88 \\
$\mathrm{p}$-valor & 0,0001 & 0,0001 & n.s. & n.s. & 0,0001 & n.s. \\
\hline
\end{tabular}

MSHj: Materia seca hoja; MSTa: Materia seca tallo; MSRz: Materia seca raíz; MS Total: Materia seca total. ns: no significante. Valores medios seguidos por diferentes letras en la misma columna difieren significativamente según el Test LSD (p<0,05).

$132 \%$ más de colonización radical que el $\mathrm{T}_{2}$. Los tratamientos $\mathrm{T}_{0} \mathrm{y} \mathrm{T}_{3}$ no presentaron micorrización. Los resultados obtenidos determinaron que la coinoculación disminuyó el porcentaje de micorrización por una acción antagónica de Th sobre el hongo micorrícico, ratificando lo informado por McGovern et al. (1992), quienes determinaron valores de micorrización de $58 \%$ en plantas de tomate cuando inocularon con Glomus intraradices. Este valor disminuyó a 22,1\% cuando las plantas se coinocularon con T. harzianum. De igual forma en plantines de tomate, Nzanza et al. (2011) obtuvieron una reducción en la micorrización de $15 \%$ después de la inoculación. La acción antagónica de $T$. harzianum se expresa parasitando el micelio y esporas de los AMF, disminuyendo así la intensidad micorrícica (Rosseau, 1996), además produce y libera metabolitos tóxicos volátiles y no volátiles a la rizosfera ejerciendo acción fungistática (Fracchia et al., 1998).

Sin embargo, los resultados de esta investigación contractan con los obtenidos por Martínez-Medina et al. (2011), quienes determinaron en plantines de melón un efecto sinérgico en la coinoculación entre Trichoderma harzianum cepa "CECT 20714" y Glomus intraradices aumentando el porcentaje de micorrización en $10 \%$.

De lo anterior se puede deducir que los hongos micorrícicos interactúan de diversas formas con los microorganismos presentes en el suelo, y esta interacción es variable según la especie de microorganismos e incluso la cepa aislada. Lo anterior coincide con Azcón-Aguilar et al. (2002), quienes señalan que los hongos micorrícicos interactúan con una amplia gama de microorganismos en la rizosfera y cuyos efectos pueden ser positivos, neutros o negativos. En esta investigación no se obtuvo un efecto positivo con la coinoculación debido a que T. harzianum tuvo un efecto detrimental sobre los niveles de micorrización en los plantines de melón.

\section{Variación poblacional de $T$. harzianum}

La población de Th (Tabla 3) no presentó diferencias estadísticas a los 50 días de postsiembra entre el tratamiento coinoculado $\left(\mathrm{T}_{2}\right)$ y el tratamiento inoculado solo con $\mathrm{Th}\left(\mathrm{T}_{3}\right)$; pero sí se obtuvieron diferencias significativas ( $\mathrm{p}$-valor $\leq 0,0001$ ) con los tratamientos no inoculados con $\mathrm{Th}\left(\mathrm{T}_{0} \mathrm{y}_{1}\right)$. También se obtuvo una disminución del crecimiento poblacional de $51,2 \%$ de UFC entre la concentración inoculada al momento de la siembra, 50 días postsiembra en ambos tratamientos $\left(\mathrm{T}_{2} \mathrm{y} \mathrm{T}_{3}\right)$; lo que se explicaría por la ocurrencia de factores abióticos asociados a la rizosfera, entre los cuales los más relevantes son períodos largos de baja humedad ambiental, $\mathrm{pH}$ básico, alta concentración de $\mathrm{CO}_{2}, \mathrm{HCO}_{3}$, sales y bajo contenido de materia orgánica (Sosa et al., 2006; Martínez et al., 2004). La fertirrigación aplicada en la etapa de almácigo en forma de $\mathrm{N}-\mathrm{NO}_{3}$ fue de $0,47 \mathrm{~g} \mathrm{~L}^{-1}$, lo que generó un efecto supresor del crecimiento de Th, coincidiendo con lo determinado por Wakelin et al. (1999), quienes determinaron mayor crecimiento de este hongo a una concentración de $0,3 \mathrm{~g} \mathrm{~L}^{-1}$ de $\mathrm{N}-\mathrm{NO}_{3}$ y una disminución significativa del crecimiento a una concentración de $0,5 \mathrm{~g} \mathrm{~L}^{-1}$ de $\mathrm{N}-\mathrm{NO}_{3}$, lo que coincide con los resultados obtenidos por Indra y Subbaiah (2003), quienes a su vez también determinaron una disminución del crecimiento del micelio y de la esporulación de Trichoderma sp. a concentraciones de $0,52 \mathrm{~g} \mathrm{~L}^{-1}$ de $\mathrm{N}-\mathrm{NO}_{3}$. 
Tabla 4. Parámetros de crecimiento en plántulas de melón Inodorus var. Honeydew a los 50 días de siembra, inoculadas con AMF y Th.

\begin{tabular}{lccccc}
\hline \multirow{2}{*}{ Tratamientos } & \multicolumn{5}{c}{ Parámetros } \\
\cline { 2 - 6 } & DT $(\mathrm{cm})$ & AP $(\mathrm{cm})$ & IA & SF $\left(\mathrm{cm}^{2}\right)$ & $\mathrm{N}^{\circ}$ de hojas \\
\hline $\mathrm{T}_{0}$ & 0,43 & 16,76 & $39,46 \mathrm{bc}$ & 80,31 & 5,6 \\
$\mathrm{~T}_{1}$ & 0,53 & 13,32 & $25,89 \mathrm{a}$ & 82,71 & 6,0 \\
$\mathrm{~T}_{2}$ & 0,50 & 14,70 & $30,69 \mathrm{ab}$ & 75,20 & 5,8 \\
$\mathrm{~T}_{3}$ & 0,45 & 19,42 & $42,46 \mathrm{c}$ & 92,42 & 5,8 \\
p-valor & n.s. & n.s. & 0,0283 & n.s. & n.s. \\
\hline
\end{tabular}

DT: Diámetro de tallo; AP: Altura de Planta; IA. Índice de ahilamiento (AP/DT); SF: Superficie foliar. n.s: no significante. Valores medios seguidos por letras iguales en la misma columna no difieren estadísticamente según el Test LSD $(\mathrm{p}>0,05)$.

La disminución poblacional de Th cuando se inoculó asociado con AMF presentó la misma tendencia que cuando se inoculó solo Th, lo que significa que el hongo micorrícico no tiene un efecto supresor sobre el crecimiento de Th y su efecto se considera neutro. Los resultados obtenidos en esta investigación son coincidentes a los obtenidos por Rodríguez et al. (2006); Martínez-Medina et al. (2004); McAllister et al. (1994) y Calvet et al. (1993).

\section{Parámetros físicos}

Los parámetros físicos como la producción de biomasa foliar, tallo y total no fueron afectados tanto por la inoculación como por la coinoculación, el efecto principal se registró en la producción de materia seca radicular (p-valor $=0,0001)($ Tabla 4$) . \mathrm{T}_{1}$ presentó la mayor producción de biomasa radicular con incrementos de 31,110 y $133 \%$ respecto de los tratamientos $\mathrm{T}_{2}, \mathrm{~T} 3 \mathrm{y} \mathrm{T}_{0}$, estos resultados determinaron que al inocular AMF la producción de raíces se vea favorecida, el efecto antagónico de Th sobre AMF se traduce en una disminución del peso seco de raíces $\left(\mathrm{T}_{2}\right)$. En los plantines inoculados solo con Th la producción de biomasa radicular no fue afectada. En general, entre los parámetros de diferenciación de crecimiento en los plantines de melón (Tabla 4) solo el índice de ahilamiento (p-valor $=0,0283)$ fue afectado significativamente por la inoculación con AMF $\left(\mathrm{T}_{1}\right)$ presentando el valor más bajo, equivalente a $35 \% 23 \%$ y $7 \%$ respecto de $\mathrm{T}_{0}$; $\mathrm{T}_{2} \mathrm{y} \mathrm{T}_{3}$, respectivamente, determinando plantines más compactos y con condiciones más aptas para el trasplante. Mwangi et al. (2009) determinaron parámetros físicos con resultados similares a los observados en almácigo de tomate, no así en esquejes de Camellia sinensis, encontrando resultados que contradicen a los de esta investigación; de igual manera, diferentes autores obtuvieron resultados neutros, sinérgicos y detrimentales. Es así como Melgar (2007) encontró un efecto neutro en plántulas de pepino, tomate y pimiento cuando fueron inoculadas con Trichoderma sp. y Glomus sp.; sin embargo, Martínez et al. (2004) y Mesa et al. (2006) determinaron un efecto detrimental en plántulas de soya y Carica papaya cuando fueron inoculadas con Gigaspora rossea y T. harzianum y G. fasciculatum y T. harzianum, respectivamente.

\section{Indicadores de estrés}

Se obtuvo una respuesta diferencial entre los indicadores de estrés evaluados, obteniéndose una mayor sensibilidad en la relación (PSRz/PSVa) (Tabla 5) respecto de la superficie foliar específica

Tabla 5. Indicadores de estrés en plántulas de melón Inodorus var. Honeydew inoculadas con AMF y Th a los 50 días de siembra.

\begin{tabular}{lcc}
\hline \multirow{2}{*}{ Tratamientos } & \multicolumn{2}{c}{ Parámetros } \\
\cline { 2 - 3 } & SFE & R(PSRz/PSVa) \\
\hline $\mathrm{T}_{0}$ & 184,84 & $0,14 \mathrm{a}$ \\
$\mathrm{T}_{1}$ & 171,09 & $0,33 \mathrm{c}$ \\
$\mathrm{T}_{2}$ & 158,68 & $0,24 \mathrm{~b}$ \\
$\mathrm{~T}_{3}$ & 174,20 & $0,13 \mathrm{a}$ \\
$\mathrm{p}$-valor & n.s. & 0,0001 \\
\hline
\end{tabular}

SFE: Superficie foliar específica (SF/Peso seco hojas); R (PSRz/PSVa): Relación (Peso seco raíz/peso seco vástago). n.s: no significante. Valores medios seguidos por diferentes letras en la misma columna difieren estadísticamente según el Test LSD $(\mathrm{p}<0,05)$. 
(SFE), donde el $\mathrm{T}_{1}$ presentó la mayor relación (PSRz/ $\mathrm{PSVa}=0,33$ ), determinado por una mayor biomasa radicular, equivalente a $135 ; 37,5$ y $153 \%$ respecto de los tratamientos $\mathrm{T}_{0}, \mathrm{~T}_{2} \mathrm{y} \mathrm{T}_{3}$, respectivamente $(\mathrm{p}$-valor $=0,0001)$.

\section{Parámetros bioquímicos}

Se obtuvieron diferencias significativas en la actividad de la NR endógena (NRE), NR inducida (NRI) y NRI/NRE (Tabla 6). $E_{n} \mathrm{~T}_{3}$ y $\mathrm{T}_{2}$ se obtuvo la mayor actividad de la NRE y NRI, respectivamente. Respecto de la relación NRI/NRE se determinaron diferencias estadísticas ( $\mathrm{p}$-valor $=0,0015)$, obteniéndose la mayor diferencia en los tratamientos $\mathrm{T}_{1} \mathrm{y}_{2}$, respecto de $\mathrm{T}_{0} \mathrm{y}_{3}$. Los resultados anteriores nos indican que el hongo micorrícico mediante el micelio extrarradical mejora la absorción de $\mathrm{N}: \mathrm{NO}_{3}$, y a la vez este nitrato es reducido a nivel de micelio externo e interno. Esto se observa en los tratamientos inoculados con AMF, los que presentan un remanente de nitratos entre 24 y $42 \%$ disponible en el tejido foliar para su reducción. Esto es confirmado por Bago et al. (1996), Govindarajulu et al. (2005) y Jin et al. (2005), quienes señalan que el micelio de los hongos micorrícicos tiene la capacidad de reducir nitrógeno nítrico al interior del micelio extrarradical y convertido en amonio, el que es transferido a la planta para la producción de proteínas. En los tratamientos sin inoculación con $\operatorname{AMF}\left(\mathrm{T}_{0}\right.$ y $\left.\mathrm{T}_{3}\right)$ se obtuvo una relación entre NRI/
$\mathrm{NRE} \leq 1$, reduciéndose todos los nitratos aportados por la fertilización ( $7 \mathrm{meq} \mathrm{L}^{-1}$ ), lo que indica que Trichoderma no tuvo ningún efecto en la absorción de nitratos y no interviene en la actividad de la enzima nitrato reductasa.

\section{Conclusiones}

La inoculación en conjunto de AMF y Trichoderma harzianum bajo las condiciones estudiadas afectó el porcentaje de colonización micorrícica en las plantas de melón, disminuyendo $57,1 \%$ respecto de las plantas inoculadas solo con el hongo micorrícico. Esta disminución determinó menor producción de biomasa radicular, afectando directamente los parámetros de crecimiento (IA) y aumentando los niveles de estrés. De igual forma la coinoculación no afectó la actividad de nitrato reductasa, parámetro que solo se vio afectado por la colonización micorrícica; por el contario, el efecto de Trichoderma sobre este parámetro fue neutro.

\section{Agradecimientos}

Los autores agradecen al Centro de Investigación y Desarrollo en Recursos Hídricos (CIDERH). CONICYT-REGIONAL R09I1001, al Departamento de Agricultura del Desierto y Biotecnología dependiente de la Universidad Arturo Prat del Estado de Chile y a la empresa BIOSIM Chile por su apoyo en esta investigación.

Tabla 6. Indicadores bioquímicos en plántulas de melón Inodorus var. Honeydew inoculadas con AMF y Th a los 50 días de siembra.

\begin{tabular}{lccc}
\hline \multirow{2}{*}{ Tratamientos } & \multicolumn{3}{c}{ Parámetros } \\
\cline { 2 - 4 } & $\mathrm{NRE}_{2}$ & $\mathrm{NRI}$ & NRI/NRE \\
\hline $\mathrm{T}_{0}$ & $1,52 \mathrm{a}$ & $1,53 \mathrm{a}$ & $1,01 \mathrm{a}$ \\
$\mathrm{T}_{1}$ & $1,29 \mathrm{a}$ & $1,58 \mathrm{a}$ & $1,24 \mathrm{bc}$ \\
$\mathrm{T}_{2}$ & $1,46 \mathrm{a}$ & $1,99 \mathrm{~b}$ & $1,42 \mathrm{bc}$ \\
$\mathrm{T}_{3}$ & $1,97 \mathrm{~b}$ & $1,53 \mathrm{a}$ & $0,78 \mathrm{a}$ \\
$\mathrm{p}$-valor & 0,0006 & 0,0068 & 0,0015 \\
\hline
\end{tabular}

NRE: Nitrato reductasa endógena; NRI: Nitrato reductasa inducida; NRI/NRE: Relación (nitrato reductasa endógena/nitrato reductasa inducida). n.s: no significante. Valores medios seguidos por diferentes letras en la misma columna difieren estadísticamente según el Test LSD $(\mathrm{p}<0,05)$. 


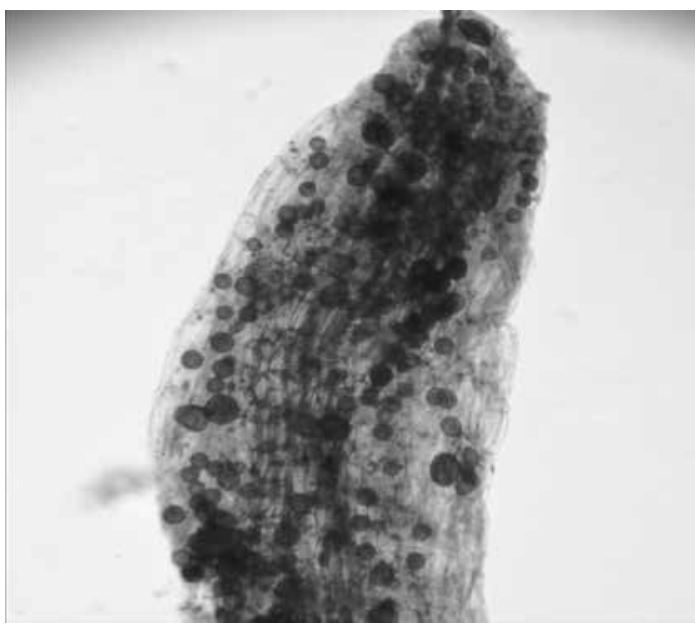

Figura 1. Raíz de melón colonizada en la que se observan esporas fúngicas intrarradicales (tratamiento $\left.\mathrm{T}_{1}\right)(40 \mathrm{X})$.

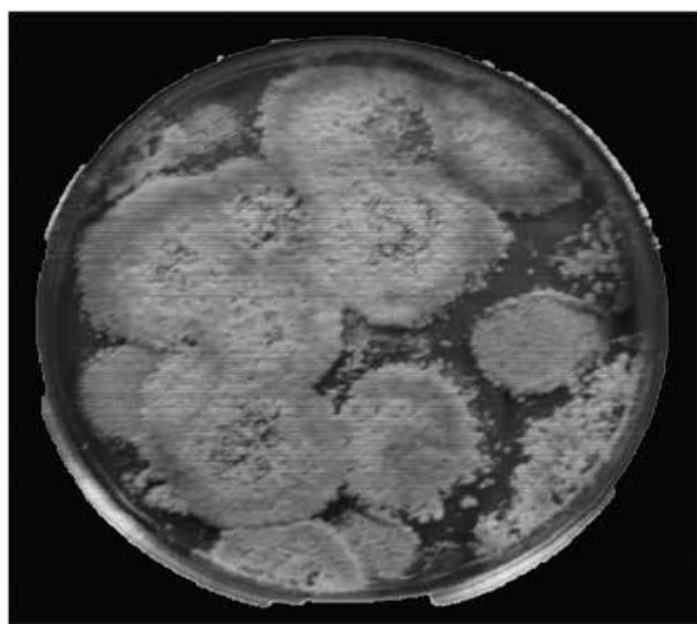

Figura 2. Unidades formadoras de colonias de Trichoderma harzianum en medio selectivo (tratamiento $\mathrm{T}_{3}$ ).

\section{Literatura Citada}

Azcón-Aguilar, C.; Palenzuela, J.; Roldán, A.; Bautista, S.; Vallejo, R.; Barea, J.M.

2002. Analysis of the mycorrhizal potential in the rizosphere of representative plant species from desertification-threatened Mediterranean shrublands. Applied Soil Ecology, 21: 1-9.

Aliye, N.; Fininsa, C.; Hiskias, Y.

2008. Evaluation of rhizosphere bacterial antagonists for their potential to bioprotect potato (Solanum tuberosum) against bacterial wilt (Ralstonia solanacearum). Biological Control, 47: 282-288.

Avis, T.; Gravel, V.; Antoun, H.; Tweddell, R.

2008. Multifaceted beneficial effects of rhizosphere microorganisms on plant health and productivity. Soil Biology \& Biochemistry, 40 (7): 1733-1740.

Bago, B.; Vierheilig, H.; Piché, Y.; Azcón-Aguilar, C.

1996. Nitrate depletion and $\mathrm{pH}$ changes induced by the extraradical mycelium of the arbuscular mycorrhizal fungus Glomus intraradices grown in monoxenic culture. New Phytologist, 133: 273-280.

Barea, J.M.; Azcón-Aguilar, C.

1982. La rizosfera: Interacciones microbio-planta. Anales de Edafología y Agrobiología, 41: 1517-1532.

Ben-Omar, N.; Merroun, M.; Arias, L.; Peñalver, J.; González,

M.; Muñoz, M.T.

1997. Comparative heavy metal biosorption study of Brewery yeast and Myxococcus xanthus biomass. Chemosphere, 10 (35): 2217-2283.

Calvet, C.; Barea, J.; Pera, J.

1993. Growth Response of Marigold (Tagetes erecta L.) to Inoculation with Glomus mosseae, Trichoderma aureoviride and Pythium ultimum in a Peat-Perlite Mixture. Plant Soil, 148: 1-6.

Chapin, F.S. III; Torn, M.S.; Tateno, M.

1996. Principles of ecosystem sustainability. American Naturalist 148: 1016-1037.
Danielson, R.; Davey, C.

1973. Non nutritional factors affecting the growth of Trichoderma in culture. Soil Biology \& Biochemistry, 5: 495-504.

Fracchia, S.; Mujica, M.; García-Romera, I.; García-Garrido,

J.; Martin, J.; Ocampo, J.; Godeas, A.

1998. Interactions between Glomus mosseae and arbuscular mycorrhizal sporocarp-associated saprophytic fungi. Plant Soil, 200: 131-137.

Green, H.; Larsen, J.; Olsson, P.A.; Jensen, D.; Jakobsen, I. 1999. Suppression of the biocontrol agent Trichoderma harzianum by mycelium of the arbuscular mycorrhizal fungus Glomus intraradices in root-free soil. Applied and Environmental Microbiology, 65: 1428-1434.

Govindarajulu, M.; Pfeffer, P.; Hairu, J.; Abubaker, J.; Douds, D.; Allen, J.W; Bücking, H.; Lammers, P.; Shachar-Hill, Y.

2005. Nitrogen transfer in the arbuscular mycorrhizal symbiosis. Nature 435: 819-823.

Guzmán, M.; Olave, J.

2006. Both electrical conductivity and sodium absorption ratio of the fertigation solution affect yield and quality of soilless melon crops. Acta Hort., (ISHS) 718: 485-490.

Harman, G.E.

2006. Overview of mechanisms and uses of Trichoderma spp. Phytopathology, 96: 190-194.

Indra, H.; Subbaiah, G.

2003. Effect of carbon and nitrogen sources on the biomass and spore production of antagonist. The Madras Agricultural Journal, 90 (7-9): 542-544.

Jin, H.; Pfeffer, P.; Douds, D.D.; Piotrowski, E.; Lammers, P.J.; Shachar-Hill, Y.

2005. The uptake, metabolism, transport and transfer of nitrogen in an arbuscular mycorrhizal symbiosis. New Phytologist, 168: 687-696. 
Martínez-Media, A.; Roldán, A.; Pascual, J.

2011. Interaction between arbuscular mycorrhizal fungi and Trichoderma harzianum under conventional and low input fertilization field condition in melon crops: Growth response and Fusarium wilt biocontrol. Applied Soil Ecology, 47: 98-105.

Martínez, A.; Obertello, M.; Pardo, A.; Ocampo, J.; Godeas, A. 2004. Interactions between Trichoderma pseudokoningii strains and the arbuscular mycorrhizal fungi Glomus mosseae and Gigaspora rosea. Mycorrhiza, 14: 79-84.

McAllister, C.B.; García-Garrido, J.M.; García-Romera, I.; Godeas, A.; Ocampo, J.A.

1994. Interaction between Alternaria alternata or Fusarium equiseti and Glomus mosseae and its effects on plant growth. Biology and Fertilizer Soils, 24, 301-305.

McGovern, R.; Datnoff, L.E.; Tripp, L.

1992. Effect of mixed infection and irrigation method on colonization of tomato roots by Trichoderma harzianum and Glomus intraradix. Florida State Horticultural Society. 105: 361-363.

Melgar, J.

2007. Evaluación del efecto de Trichoderma sp. y Glomus sp. en la incidencia y severidad de enfermedades del suelo y en el rendimiento de tomate, chile dulce y pepino. FHIAComayagua. 12: 1-3.

Mesa, J.; Gómez, J.; Rodríguez, O.; Parets, E.; Soto, R. 2006. Efecto de Trichoderma y micorrizas en la producción de posturas de Carica papaya L. Centro Agrícola, 3: 75-81.

Mwangi, M.; Monda, E.; Okoth, S.; Jefwa, J.

2009. Effect of Trichoderma harzianum and arbuscular mycorrhizal fungi on growth in tomato (Lycopersicum esculentum mill) seedlings, napier (Pennisetum purpureum) and tea (Camellia sinensis) cuttings. Tropical and Subtropical Agroecosystems, 11: 423-429.

Nzanza, B.; Marais, D.; Soundy, P.

2011. Tomato (Solanum lycopersicum L.) seedling growth and development as influenced by Trichoderma harzianum and arbuscular mycorrhizal fungi. African Journal of Microbiology Research, 5 (4): 425-431.

Paul, E.A.; Clark, F.E.

1989. Soil microbiology and biochemistry. Academic Press, San Diego. 273 pp.

Philips, J.; Hayman, D.

1970. Improved procedures for clearing roots and staining parasitic and vesicular arbuscular mycorrhizal fungi for rapid assessment of infection. Transactions of the British Mycological Society. 55: 158-161.

Rabeendran, N.; Moot, D.; Jones, E.; Stewart, A.

2000. Inconsistent growth promotion of cabbage and lettuce from Trichoderma isolates. New Zeland Plant Protection. 53, 143-146.

Rodríguez, T.; Sánchez, J.; Morales, E.; Cruz, F.

2006. Interacción micorrizas arbusculares-Trichoderma harzianum (moniliaceae) y efectos sobre el crecimiento de Brachiaria decumbens (poaceae). Acta Biológica Colombiana, 11 (1): 43-54.

Rousseau, A.

1996. Mycoparasitism of the Extramatrical Phase of Glomus intraradices by Trichoderma harzianum. Phytopathology, 86 (5): 434-443.

Sosa, T.; Sánchez, J.; Morales, E.; Cruz, F.

2006. Interacción micorrizas arbusculares-Trichoderma harzianum (Moniliaceae) y efectos sobre el crecimiento de Brachiaria decumbens (Poaceae). Acta Biológica Colombiana. 11 (1): 43-54.

Trouvelot, A.; Kough, J.L.; Gianinazzi-Pearson, V.

1986. Mesure du taux de mycorrhization VA d'un système radiculaire. Recherche de methods d'estimation ayantune signification function elle. En: "Physiological and genetical aspects of mycorrhizae". Gianinazzi-Pearson, V. and Gianinazzi, S. eds., INRA, Paris, 101-109.

Urrestarazu, M.

2004. Tratado de cultivo sin suelo. Ediciones Mundi-Prensa. $914 \mathrm{p}$.

Wakelin, S.; Sivasithamparam, K.; Cole, A.; Skipp, R.

1999. Saprophytic growth in soil of a strain of Trichoderma koningii. New Zeland. Journal of Agricultural Research, 42: 37-345.

Williams, J.; Clarkson, J.M.; Mills, P.; Cooper, R.

2003. A selective medium for quantitative reisolation of Trichoderma harzianum from Agaricus bisporus compost. Applied and Environmental Microbiology, 69 (7): 4190-4191.

Valenzuela, J.L.; Sánchez, A.; Romero, L.

1987. Assesing critical N supply by means of nitrate-reductase activity in tomato and cucumber plants. Journal of Plant Nutrition, 10: 1733-1741.

Yasir, M.; Aslam, Z.; Kim, S.W.; Lee, S.W.; Jeon, C.O.; Chung, Y.R. 2009. Bacterial community composition and chitinase gene diversity of vermicompost with antifungal activity. Bioresource Technology, 100: 4396-4403. 